\title{
Functional Neuromuscular Stimulation Controlled by Surface Electromyographic Signals Produced by Volitional Activation of the Same Muscle Adaptive Removal of the Muscle Response from the Recorded EMG-Signal
}

Sennels, Søren; Biering-Sørensen, Fin; Andersen, Ole Trier; Hansen, Steffen Duus

Published in:

IEEE Transactions on Rehabilitation Engineering

Link to article, DOI:

$10.1109 / 86.593293$

Publication date:

1997

Document Version

Publisher's PDF, also known as Version of record

Link back to DTU Orbit

Citation (APA):

Sennels, S., Biering-Sørensen, F., Andersen, O. T., \& Hansen, S. D. (1997). Functional Neuromuscular Stimulation Controlled by Surface Electromyographic Signals Produced by Volitional Activation of the Same Muscle: Adaptive Removal of the Muscle Response from the Recorded EMG-Signal. IEEE Transactions on Rehabilitation Engineering, 5(2), 195-206. https://doi.org/10.1109/86.593293

\section{General rights}

Copyright and moral rights for the publications made accessible in the public portal are retained by the authors and/or other copyright owners and it is a condition of accessing publications that users recognise and abide by the legal requirements associated with these rights.

- Users may download and print one copy of any publication from the public portal for the purpose of private study or research.

- You may not further distribute the material or use it for any profit-making activity or commercial gain

- You may freely distribute the URL identifying the publication in the public portal 


\title{
Functional Neuromuscular Stimulation Controlled by Surface Electromyographic Signals Produced by Volitional Activation of the Same Muscle: Adaptive Removal of the Muscle Response from the Recorded EMG-Signal
}

\author{
Søren Sennels, Fin Biering-Sørensen, Ole Trier Andersen, \\ and Steffen Duus Hansen, Member, IEEE
}

\begin{abstract}
In order to use the volitional electromyography (EMG) as a control signal for the stimulation of the same muscle, it is necessary to eliminate the stimulation artifacts and the muscle responses caused by the stimulation. The stimulation artifacts, caused by the electric field in skin and tissue generated by the stimulation current, are relatively easy to eliminate by shutting down the EMG-amplifier at the onset of the stimulation pulses. The muscle response is a nonstationary signal, therefore, an adaptive linear prediction filter is proposed. The filter is implemented and for three filter lengths tested on both simulated and real data. The filter performance is compared with a conventional fixed comb filter. The simulations indicate that the adaptive filter is relatively insensitive to variations in amplitude of the muscle responses, and for all filter lengths produces a good filtering. For variations in shape of the muscle responses and for real data, an increased filter performance can be achieved by increasing the filter length. Using a filter length of up to seven stimulation periods, it is possible to reduce real muscle responses to a level comparable with the background noise. Using the shut-down circuit and the adaptive filter both the stimulation artifacts and the muscle responses can be effectively eliminated from the EMG signal from a stimulated muscle. It is therefore possible to extract the volitional EMG from a partly paralyzed muscle and use it for controlling the stimulation of the same muscle.
\end{abstract}

Index Terms-Adaptive filters, EMG-control, functional electrical stimulation, muscle response, spinal cord injury, tetraplegia.

\section{INTRODUCTION}

$\mathbf{I}$ NDIVIDUALS who have suffered from a complete spinal cord injury (SCI) at C5-C6 cervical level have no hand function left, whereas limited reaching is often still possible by using the shoulder muscles and elbow flexors. Functional electrical stimulation (FES) gives the possibility of restoring

Manuscript received July 2, 1996; revised February 25, 1997. This work was supported by the Danish Technical Research Council and the Danish Medical Research Council.

S. Sennels and S. D. Hansen are with the Department of Mathematical Modeling, Technical University of Denmark, DK-2800 Lyngby, Denmark.

F. Biering-Sørensen is with the Center for the Spinal Cord Injured, University Hospital of Copenhagen, Denmark.

O. T. Andersen is with the Department of Information Technology, Technical University of Denmark, DK-2800 Lyngby, Denmark.

Publisher Item Identifier S 1063-6528(97)04420-0. some of the lost hand function by activation of the paralyzed muscles by electrical current pulses. Especially restoring a hand grasp will be a major benefit for these individuals and improve their possibilities in performing daily activities. Restoration of hand grasping by FES has been done with surface stimulation [1], [2], percutaneous electrodes [3], [4] and with fully implanted electrodes [5].

Using surface stimulation both the BIONIC Glove [2] and the Handmaster system [6] provides a lateral pinch grip. This pinch grip is established by stimulating the finger and thumb flexor muscles, and hand opening is provided by stimulating the wrist and finger extensors.

The common control strategy is to enable the user to modify the stimulation intensity applied to the paralyzed muscles by activating a motor function which is under voluntary control. The control implementations proposed in literature are numerous. Mechanical systems have been proposed, where the user uses a motor function under voluntary control to handle a joystick [2], [7]. For C4 tetraplegics, who have only very limited motor function, a voice controlled system [1] and a respiration controlled system [3] have been proposed.

As an alternative to the mechanical control systems, several authors have proposed to use the EMG-signal for controlling the neural prosthesis [4], [8]-[15]. Several requirements must be fulfilled for using the EMG as a control signal:

- the control muscle must be under at least some voluntary control;

- the control muscle should not be active during other normal upper extremity functions and thereby trigger unwanted stimulation;

- the stimulation should not interfere with the recorded signal.

These requirements have often resulted in systems where the EMG from proximal muscles has been used to stimulate more distal muscles. For example, the sternocleidomastoid muscles have been used to control hand opening and closing, since they are easy to activate and they do not have an increased activity during grasping and reaching in tetraplegic individuals [4], 


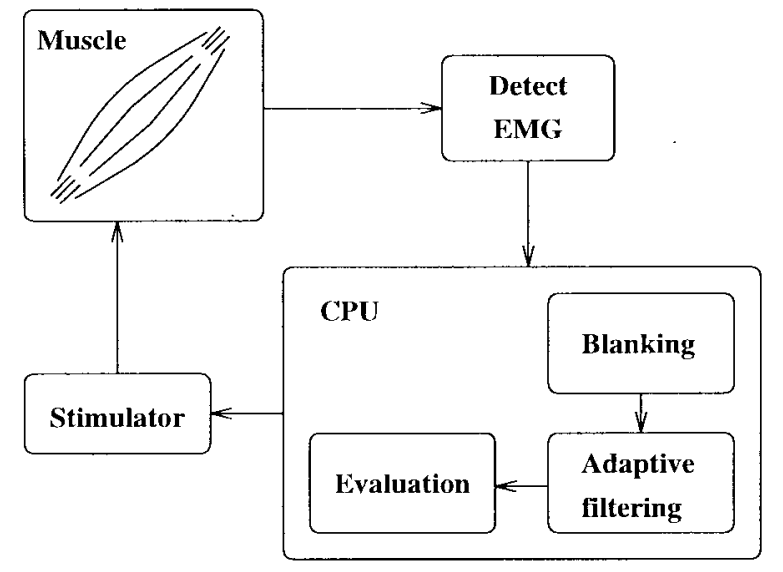

Fig. 1. Block diagram of the control strategy. The EMG is detected by surface electrodes from a stimulated partly paralyzed muscle. By processing it through a blanking circuit and an adaptive filter the stimulation generated artifacts and muscle responses are eliminated and a residual signal can be extracted. This signal is related to the volitional EMG and can be used to determine the stimulation intensity applied to the same muscle.

[15]. But also the trapezius muscle has been used to control the stimulation of the opposite forearm [8].

By eliminating the influence from the stimulation on the recorded signal it is possible to use the EMG from muscles located near to the stimulated muscles. For example the EMG from the wrist extensor muscles has been used to control the stimulation of the finger and thumb flexors in order to obtain a stronger tenodesis grip [14].

The end goal of this study is to establish a lateral pinch grip (or key grip) in C5-C6 tetraplegics using surface stimulation. This can be achieved by a wrist extension by stimulating $\mathrm{mm}$. extensor carpi radialis longus/brevis and making use of the tenodesis effect. The tenodesis effect is a passive finger flexion and thumb adduction, due to the shortening of the tendons caused by the extension of the wrist. The lateral pinch grip can thus be established by stimulating only one muscle. Since the tenodesis grip is a passive grasp, additional grasp force can be generated by also stimulating the thumb flexors.

The wrist extensor muscles are innervated from the fifth-seventh cervical spinal cord segments, a C5-C6 lesion will therefore often result in only partly paralyzed wrist extensor muscles. The individuals will typically have some voluntary control of these muscles, but usually not enough to provide a function or even to perform wrist movements. Still the volitional EMG activity can be detected and used for controlling the stimulation of the same muscles as illustrated in Fig. 1. The idea is to record the EMG signal from the stimulated muscle and to extract a residual signal, which can be interpreted as a desire for a certain muscle contraction. Appropriate stimulation parameters can then be calculated and applied to the same partly paralyzed muscle. In this way an EMG-controlled lateral pinch grip can be obtained, which can be used for activities of daily living.

This control strategy has several advantages.

- Since the user activates the same muscles as being stimulated, it might result in a natural way for the user to control his/her neural prosthesis.

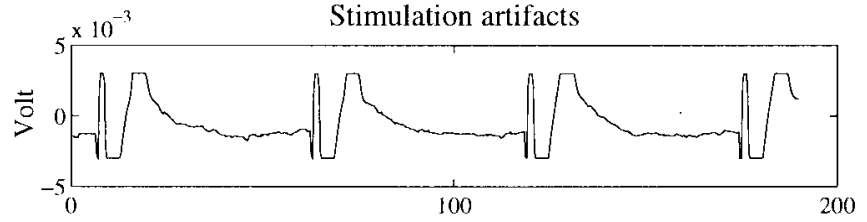

(a)

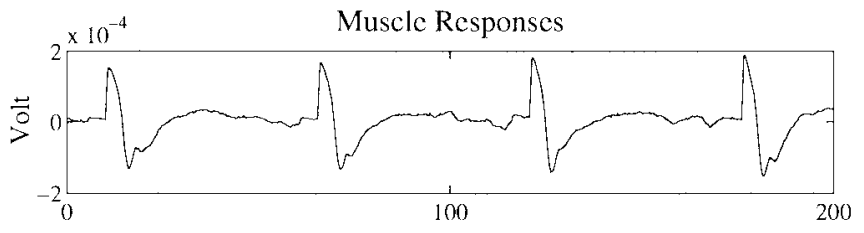

(b)

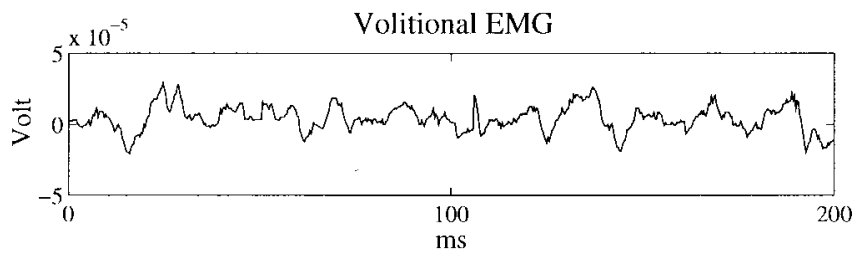

(c)

Fig. 2. Typical time courses of the signal components in EMG-recordings from the wrist extensor muscles of a tetraplegic individual. (a) EMG-recording during $20 \mathrm{~Hz}$ stimulation of the wrist extensor muscles with no volitional muscle activity. This signal is dominated by stimulation artifacts. The amplifier is saturated and does not fully recover during the interpulse interval. (b) Blanked EMG-recording during $20 \mathrm{~Hz}$ stimulation of the wrist extensor muscles with no volitional activity. This signal is dominated by muscle responses. (c) EMG from the volitional activated wrist extensor muscles with no stimulation applied.

- There is no requirement to the degree of voluntary muscle force the patient must generate. Only that some voluntary control persist.

- No mechanical transducer has to be mounted and in a future version the recording and stimulating electrodes can be the same. This might ease mounting of the system and make the final design more cosmetically acceptable.

Two types of artifacts usually exist in the EMG-signal from an electrically stimulated muscle as illustrated on Fig. 2. Stimulation artifacts and muscle responses. A typical time course of an EMG-signal dominated by stimulation artifacts is shown in Fig. 2(a). The stimulation artifacts are due to the electric field in the tissue and skin generated by the stimulation current. In Fig. 2(b) is shown a typical time course of an EMG-recording, where the stimulation artifacts has been removed by a blanking circuit. This signal is dominated by muscle responses (or M-waves). The muscle response is due to the simultaneous activation of many motor units caused by the stimulation. For comparison a typical time course of the voltional EMG from a tetraplegic individual is shown in Fig. 2(c). The magnitude of the stimulation artifacts can be more than a magnitude larger than the muscle response, which again can be more than a magnitude larger than the volitional EMG.

This paper focus on the task of removing the artifacts evoked by the stimulation in the recorded EMG-signal in order 


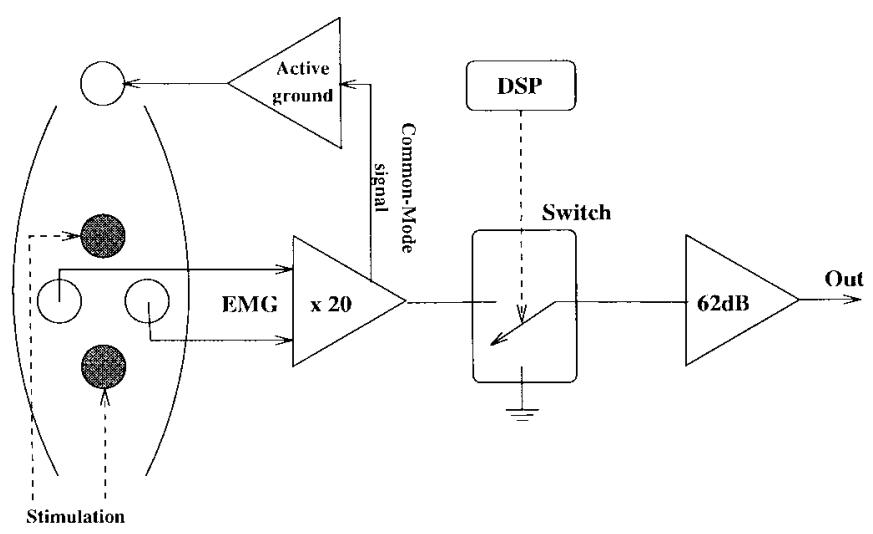

Fig. 3. Differential EMG-amplifier with shut-down control for elimination of stimulation artifacts. The switch is controlled by the signal processor and the blanking time can be set by the operator. The total amplifier has an adjustable gain of up to approximately $88 \mathrm{~dB}$ and $3 \mathrm{~dB}$ corner frequencies 10 and 1500 $\mathrm{Hz}$.

to extract a residual signal, which can be related to the volitional muscle activity and used for controlling the stimulation. The stimulation artifacts are eliminated by an analog blanking circuit, whereas a well-known adaptive filtering technique is proposed for the suppression of the muscle responses.

\section{METHODS}

The control strategy illustrated in Fig. 1 has been implemented in a system consisting of an EMG-amplifier with a blanking circuit, a constant-current stimulator and a user interface. The system is build up around an ADSP-21020 digital signal processor, which handles the signal processing and controls both the blanking circuit and the stimulator [16].

\section{A. Eliminating the Stimulation Artifact}

The stimulation artifacts can effectively be eliminated by shutting down the amplifier at the onset of the stimulation pulses. This principle has been demonstrated previously [14], [17]. Therefore an EMG-amplifier with a switch system was implemented as illustrated on Fig. 3. Notice, that the stimulation electrodes are placed along the muscle fiber direction to increase selectivity, and the recording electrodes are placed symmetrically around these across the muscle. This electrode configuration will assist in suppression the stimulation artifacts, due to the differential recording technique [13]. The recorded EMG signals may therefore also contain contributions from other muscles and do not reflect the same physiological parameters as if the electrodes were aligned along the fibers. This electrode configuration is the only practical possibility in the present system. But in future versions, the recording and stimulating electrodes will be the same and aligned along the fiber direction.

The input stage of the EMG-amplifier consists of a differential amplifier with FET-transistors at the input with a gain of approximately 20 times and with an active ground system, which forces the common mode signal back to the patient.
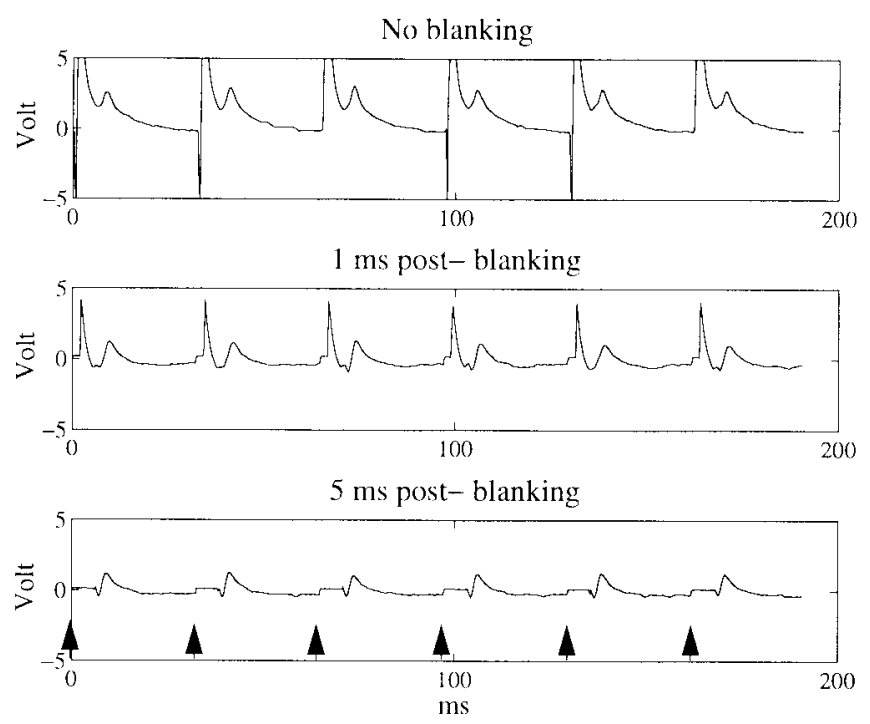

Fig. 4. Example of the effect of the shut-down circuit with the blanking time extended 1 and $5 \mathrm{~ms}$ after the end of the stimulation pulse. The 30 $\mathrm{Hz}$ constant-current stimulations were applied to the relaxed wrist extensor muscles of a tetraplegic individual. The arrows indicate the start of the stimulation pulse. The long transients in the top figure are due to saturation of the amplifier.

This reduces $50 \mathrm{~Hz}$ noise and increases the overall commonmode rejection ratio $(>80 \mathrm{~dB})$. The input stage is followed by the switch configuration, which connects the output stage to ground at the onset of the stimulation pulses. The switch is controlled by the signal processor and the blanking time is adjustable by the operator. In order to reduce switching noise a Bi-CMOS switch (PC74/HCT4053) is used due to its short switching time (typically, 23 ns.) and low parasitic capacities $(<8 \mathrm{pF})$. It is possible to reduce the switching noise to a level comparable with the background noise which is approximately $4 \mu \mathrm{V}$ measured at the electrodes. The overall amplifier has an adjustable gain of up to approximately $88 \mathrm{~dB}$ and $3 \mathrm{~dB}$ corner frequencies 10 and $1500 \mathrm{~Hz}$.

Fig. 4 shows an example of the effect of the shut-down circuit where the blanking is extended 1 and $5 \mathrm{~ms}$ after the end of the stimulation pulses. Both blanking times are capable of removing enough of the stimulation artifacts to avoid overloading of the amplifier. Increasing the blanking time will further reduce both the amount of stimulation artifacts and muscle responses. In order to obtain as many samples as possible containing information of the volitional EMG, it is desirable to have a short blanking time. The blanking time should therefore be as short as possible, but still large enough to ensure that the amplifier is not overloaded. The optimal blanking time will typical depend on the stimulation intensity and electrode position, and in a practical situation it should be set with a proper margin to avoid overload.

\section{B. Suppression of Muscle Responses}

The muscle response can in some cases extend for an entire stimulation period especially when high-stimulation frequencies are used. Therefore it can not be eliminated only by extending the blanking time. Some authors have assumed 


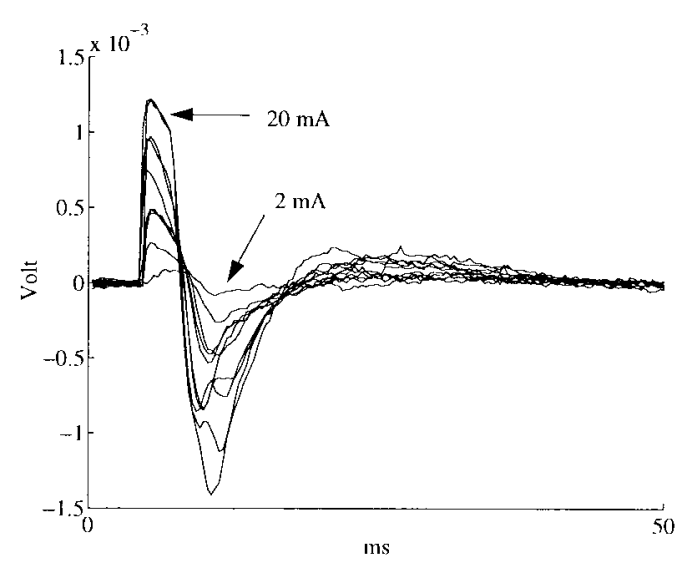

Fig. 5. Typical muscle responses obtained from a tetraplegic individual with $20 \mathrm{~Hz}$ constant-current stimulation applied to the wrist extensor muscles. The stimulation amplitude were linearly increased from 0 to $20 \mathrm{~mA}$ over a duration of $1.5 \mathrm{~s}$. For each curve is the stimulation intensity increased by $2 \mathrm{~mA}$. The values on the ordinate is calculated with respect to the input side of the amplifier.

the muscle responses to be stationary and therefore use fixed comb filters [12], [13]. But the muscle response is clearly a nonstationary signal, since it depends on many factors, such as: Stimulation intensity, position of both stimulating and recording electrodes, limb position, fatigue, the contraction level of the muscle, etc. (see [18]). Therefore, an adaptive filter solution is proposed based on observations of the muscle responses. Typical recorded muscle responses from a tetraplegic individual where $20 \mathrm{~Hz}$ stimulation was applied with linear increasing amplitude until maximum contraction, are shown in Fig. 5.

By observing a number of consecutive muscle responses, within a time window of less than $500 \mathrm{~ms}$, two observations can be noted.

- The amplitude of the muscle responses can exhibit relatively large variations from one stimulation period to another. Due to the steep recruitment curve only moderate changes in stimulation amplitude can cause large variations in the amplitude of the muscle responses. The rate can be more than 0.2 V/A measured at the electrodes.

- The shape of the muscle responses exhibits only moderate and relative slow variations within the observed time window. The variations in shape are mainly due to the relatively slow limb movements and to changes in the contraction level of the muscle.

Due to modulation of the stimulation amplitude, the variations in amplitude of the muscle responses are relatively fast compared to the variations in shape. A well-known adaptive linear prediction filter ${ }^{1}$ [19], which is insensitive to these variations in amplitude, is therefore proposed as illustrated in Fig. 6.

The filtering idea is to divide the input signal, $x(i)$, into $M+1$ frames each consisting of $N$-samples. By choosing $N$ as the ratio of the sampling frequency to the stimulation frequency it can be obtained that the muscle responses are aligned identically in each frame. The present frame is then

\footnotetext{
${ }^{1}$ The filter uses a block-wise adaption method instead of a gradient method.
}

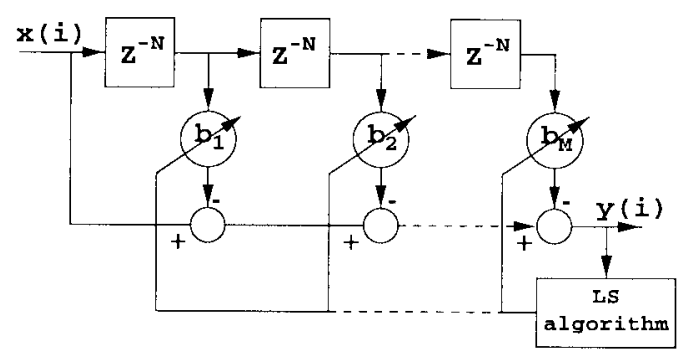

Fig. 6. Linear adaptive prediction filter for suppression of muscle responses. The input to the filter is the blanked EMG signal. $N$ is the ratio of the sampling frequency to the stimulation frequency. $M$ is the filter length.

predicted from a linear combination of $M$ foregoing frames. If the prediction is good enough, subtracting the predicted frame from the present frame will leave a residual signal where the muscle response has been eliminated. Each time $N$ samples have been recorded, the optimal filter coefficients are determined and $N$ output samples are calculated. The filter thus adapts to the signal variations at a rate equal to the stimulation frequency (typically, 20-30 Hz).

In the following it is assumed that the input signal $x(i)$ can be written as a sum of the muscle responses and the volitional EMG: $x(i)=m(i)+v(i)$. The volitional EMG is assumed to be a band-limited Gaussian signal. This is a reasonable approximation, when the number of active motor units exceeds 15 [20]. (See Section IV for further discussion.)

The output of the filter is given by

$$
y(i)=x(i)-\sum_{j=1}^{M} b_{j} x(i-j N)
$$

Letting $\mathbf{s}_{j}=[x(-(j+1) N+1) \cdots x(-j N)]$ denote the vector containing the $N$ samples of the $j$ th previous recorded frame, the predicted present output frame can then be written as

$$
\mathbf{y}_{0}=\mathbf{s}_{0}-\sum_{j=1}^{M} b_{j} \mathbf{s}_{j} .
$$

If an optimal filtering is performed and all of the muscle responses are eliminated, the power of the present output frame will have its minimum value. Notice that only the samples in the interval $[-(M+1) N+1 ; 0]$ are considered, whereas no assumptions are made about the data outside this interval. This is also known as the covariance method for data windowing [19]. The optimal filter coefficients are updated after each frame (or after $N$ samples) and can be found by a usual leastsquare algorithm [19] where the output energy of the present frame is minimized with respect to the filter coefficients

$$
\frac{d}{d b_{j}}\left(\sum_{i=-N+1}^{0} y(i)^{2}\right)=\frac{d}{d b_{j}}\left(\mathbf{y}_{0} \mathbf{y}_{0}^{T}\right)=0, \text { for } j=1 \cdots M
$$

It can be shown [19] that this function has only one minimum and no maxima. The least-square algorithm (3) will therefore always give an unique optimal solution. 
The output energy of the present frame is given by

$$
\begin{aligned}
\sum_{i=-N+1}^{0} y(i)^{2}= & \mathbf{y}_{0} \mathbf{y}_{0}^{T}=\left(\mathbf{s}_{0} \sum_{j=1}^{M} b_{j} \mathbf{s}_{j}\right)\left(\mathbf{s}_{0}-\sum_{j=1}^{M} b_{j} \mathbf{s}_{j}\right)^{T} \\
= & \mathbf{s}_{0} \mathbf{s}_{0}^{T}-2 \sum_{j=1}^{M} b_{j} \mathbf{s}_{0} \mathbf{s}_{j}^{T}+\sum_{j=1}^{M} \sum_{k=1}^{M} b_{j} b_{k} \mathbf{s}_{j} \mathbf{s}_{k}^{T} \\
= & \mathbf{s}_{0} \mathbf{s}_{0}^{T}-2 \sum_{j=1}^{M} b_{j} \mathbf{s}_{0} \mathbf{s}_{j}^{T} \\
& +\sum_{j=1}^{M} b_{j}^{2} \mathbf{s}_{j} \mathbf{s}_{j}^{T}+\sum_{j=1}^{M} \sum_{\substack{k=1 \\
k \neq j}}^{M} b_{j} b_{k} \mathbf{s}_{j} \mathbf{s}_{k}^{T} .
\end{aligned}
$$

Minimizing this function with respect to the $l$ th filter coefficient gives

$$
\begin{aligned}
& \frac{d}{d b_{l}}\left(\sum_{i=-N+1}^{0} y(i)^{2}\right)=-2 \mathbf{s}_{0} \mathbf{s}_{l}^{T}+2 b_{l} \mathbf{s}_{l} \mathbf{s}_{l}^{T} \\
& +2 \sum_{\substack{j=1 \\
j \neq l}}^{M} b_{j} \mathrm{~s}_{j} \mathrm{~s}_{l}^{T}=0, \quad \text { for } l=1 \cdots M \\
& \Uparrow \\
& \sum_{j=1}^{M} b_{j} \mathbf{s}_{j} \mathbf{s}_{l}^{T}=\mathbf{s}_{0} \mathbf{s}_{l}^{T}, \quad \text { for } l=1 \cdots M
\end{aligned}
$$
form

Equation (6) can be written more conveniently on matrix

$$
\mathbf{\Phi b}=\Theta \quad \Leftrightarrow \quad \mathbf{b}=\boldsymbol{\Phi}^{-1} \Theta
$$

where

$$
\begin{aligned}
\boldsymbol{\Phi} & =\left[\begin{array}{cccc}
\phi_{1,1} & \phi_{1,2} & \cdots & \phi_{1, M} \\
\phi_{1,2} & \phi_{2,2} & \cdots & \phi_{2, M} \\
\vdots & \vdots & \ddots & \vdots \\
\phi_{1, M} & \phi_{2, M} & \cdots & \phi_{M, M}
\end{array}\right] \\
\boldsymbol{\Theta} & =\left[\phi_{0,1}, \phi_{0,2}, \cdots, \phi_{0, M}\right]^{T} \\
\mathbf{b} & =\left[b_{1}, b_{2}, \cdots, b_{M}\right]^{T} \\
\phi_{r, s} & =\mathbf{s}_{r} \mathbf{s}_{s}^{T}=\sum_{i=-N+1}^{0} x(i-r N) x(i-s N) .
\end{aligned}
$$

Notice that (7) has the same structure as a the normal equations for a linear least-square filter using the covariance method for data windowing. It just operates on vectors instead of single samples [19].

Assuming that the filter removes all the muscle responses and that the volitional EMG is band-limited Gaussian noise, the power of the output signal is given by

$$
P_{\text {out }}=P_{0}+\sum_{j=1}^{M} b_{j}^{2} P_{j}
$$

where $P_{j}$ is the power of the volitional EMG in the $j$ th frame. It is assumed that the power of the volitional EMG is constant in the $M+1$ frames. A proper scaling of the output signal will then result in a residual signal where the power is independent of the filter coefficients and with the same rms-value as the volitional EMG

$$
\tilde{y}(i)=\frac{y(i)}{\sqrt{1+\sum_{j=1}^{M} b_{j}^{2}}} .
$$

The normal equations (7) for $M$ up to six are solved in real time on a ADSP-21020 digital signal processor. A LUdecomposition is performed using Crout's method followed by forward- and backsubstitution [21].

\section{Simulated Data}

When recording the EMG from a stimulated muscle it is not possible prior to the filtering to separate the signal components generated by the stimulation and the components generated by the voluntary muscle contraction. Therefore, when evaluating the filter performance, the evaluation will depend not only of the filter performance, but also on the power of both the muscle responses and the volitional EMG. It is therefore not possible, using real EMG-recordings, to compare the filter performance for different input signals. Using simulated data it is possible to calculate a filter performance measure, which only reflects the performance of the filter.

The variations in the muscle responses during an increase in stimulation amplitude could be described as a variation in amplitude and a variation in shape (see Fig. 5). Based on observations from a number of real muscle responses, it was found that a real muscle response could be simulated as an exponentially damped sinusoid, where a scaling parameter simulates variations in amplitude and exponential time constant simulates variations in shape. The volitional EMG was simulated as additive band-limited Gaussian noise $\left(\mu=0, \sigma^{2}=1\right)$. The $j$ th frame of the recorded EMG was thus simulated as

$$
s_{j}(i)=a_{j} e^{-i / \tau_{j}} \sin (2 \pi 3 i / N)+v(i), \text { for } i=0 \cdots N-1
$$

$N$ was set to 111 samples corresponding to a sampling frequency of $3.333 \mathrm{kHz}$ and a stimulation frequency of 30 $\mathrm{Hz}$. The parameter, $a_{j}$, is a scaling parameter determining the amplitude of the $j$ th muscle response and $\tau_{j}$ is a parameter determining the shape. Choosing $a=200$ and $\tau=20$ gives a power ratio of the noise signal to the simulated muscle response of approximately $-32 \mathrm{~dB}$ and also gives a simulated muscle response which is significantly larger than the noise signal in the entire stimulation period. Compared to real signals, these values can be regarded as a worst case situation. To simulate variations in amplitude and shape of the muscle responses, both parameters were set to a new value at the onset of each frame. The new values were calculated as a random number in a symmetrical range around the fixed value.

Both the pure noise signal, $v(i)$, and the mixed signal, $s(i)$, were processed through the filters and a muscle response index (MRI) was defined and calculated both at the input $\left(\mathrm{MRI}_{x}\right)$ and 
at the output $\left(\mathrm{MRI}_{y}\right)$ of the filter

$$
\begin{aligned}
\operatorname{MRI}_{x} & =10 \log \left(\frac{\sum v_{x}(i)^{2}}{\sum s_{x}(i)^{2}}\right) \\
\operatorname{MRI}_{y} & =10 \log \left(\frac{\sum v_{y}(i)^{2}}{\sum s_{y}(i)^{2}}\right)
\end{aligned}
$$

where index $x$ and $y$ denote the signal at the input, respectively, at the output of the filter.

When a perfect filtering is performed and all of the muscle responses are eliminated, $s_{y}(i)$ will be a Gaussian signal with the same power as $v_{y}(i)$. A perfect filtering will therefore result in a $\mathrm{MRI}_{y}$ of $0 \mathrm{~dB}$.

\section{Real Data}

The filters were also tested on real muscle responses obtained from the wrist extensor muscles of two tetraplegic individuals and one healthy individual. During a normal grasping task, the muscle responses will mainly be modulated due to variations in the stimulation amplitude and due to limb movements as the electrodes are displaced relative to the muscle and the muscle properties change. The filters should therefore be tested during modulations in stimulation current and during limb movements. The ladder was not practical possible in the two tetraplegic individuals. These measurements were therefore carried out on a healthy individual. The first tetraplegic individual (SCI 1) had an incomplete C5lesion, whereas the second tetraplegic individual (SCI 2) had a complete C5-lesion. Both individuals had wrist extensor muscles of strength 2 .

From each individual a set of input signals were generated and processed through each of the filters. Biphasic stimulation was applied to the $\mathrm{m}$. extensor carpi radialis brevis through two $30 \mathrm{~mm}$ diameter round shape felt pad electrodes (Jožef Stefan Institute, Slovenia). The electrodes were soaked in plain water and mounted with velcro straps along the muscle. The maximum stimulation intensity was set to give a full extension of the wrist. The EMG signals were recorded using three disposable, self-adhering ECG-electrodes (Blue Sensor, MEDICOTEST A/S, Denmark) with $1 \mathrm{~cm}$ diameter round shape recording area. Electrode paste was used to establish a good skin contact. The two recording electrodes were mounted across the muscle, symmetrical to the stimulating electrodes (cf., Fig. 3). The ground electrode was positioned arbitrarily on the wrist. The EMG-signals were recorded and blanked with the EMG-amplifier (Fig. 3) with a post blank time of 5 $\mathrm{ms}$. The gain was adjusted to give a maximum output voltage of $\pm 5 \mathrm{~V}$ and the output was sampled at $3.333 \mathrm{kHz}$ using an analog and digital I/O-board (DT2811, Data Translation Inc., $\mathrm{MA}$,) in a conventional IBM-compatible PC. The filterings were performed in MATLAB.

For all three individuals, data sets were generated with stimulation applied as a $3 \mathrm{~s}$ increasing ramp followed by a 3 s decreasing ramp. From the healthy individual also data sets were generated during forearm movements. Three forearm movements were chosen arbitrarily: an elbow flexion from fully extended to fully flexed elbow, forearm supination from a position with the palm of the hand on the table to fully
TABLE I

Muscle Response Index (MRI) for Simulated Data Processed by a Fixed Coefficient Filter (Fixed) and Adaptive Filters of LengthS: $M=1,3$ AND 6 . The PARAMETERS $a$ AND $\tau$ Determine the Variation in Amplitude, Respectively, Shape of the Simulated Muscle Responses. Both $a$ AND $\tau$ EXHIBIT Random Variations Within a Symmetrical Range Around a Fixed Value

\begin{tabular}{rc|c|rrrr}
\hline & & MRI $_{x}$ & \multicolumn{4}{|c}{ MRI $_{y}$} \\
\hline \multicolumn{1}{c}{} & $\tau$ & {$[\mathrm{dB}]$} & Fixed & $\mathrm{M}=1$ & $\mathrm{M}=3$ & $\mathrm{M}=6$ \\
\hline $200 \pm 0 \%$ & $20 \pm 0 \%$ & -32.8 & 0.0 & 0.1 & -0.1 & -0.2 \\
\hline $200 \pm 10 \%$ & $20 \pm 0 \%$ & -32.9 & -9.8 & -0.3 & -0.2 & -0.3 \\
$200 \pm 50 \%$ & $20 \pm 0 \%$ & -33.0 & -23.3 & -2.9 & -1.4 & -0.5 \\
$200 \pm 100 \%$ & $20 \pm 0 \%$ & -33.2 & -28.9 & -5.8 & -1.5 & -0.4 \\
\hline $200 \pm 0 \%$ & $20 \pm 10 \%$ & -32.9 & -8.1 & -3.9 & -0.9 & -0.4 \\
$200 \pm 0 \%$ & $20 \pm 20 \%$ & -32.8 & -12.4 & -7.3 & -0.5 & -0.1 \\
$200 \pm 0 \%$ & $20 \pm 40 \%$ & -33.5 & -17.8 & -11.4 & -0.7 & -0.3 \\
$200 \pm 0 \%$ & $20 \pm 60 \%$ & -32.6 & -23.5 & -16.6 & -2.7 & -1.0 \\
$200 \pm 0 \%$ & $20 \pm 80 \%$ & -32.7 & -23.1 & -15.8 & -1.7 & -1.4 \\
$200 \pm 0 \%$ & $20 \pm 100 \%$ & -32.7 & -28.9 & -19.8 & -4.8 & -2.6 \\
\hline $200 \pm 50 \%$ & $20 \pm 40 \%$ & -31.3 & -23.4 & -11.1 & -2.0 & -0.8 \\
$200 \pm 100 \%$ & $20 \pm 100 \%$ & -33.2 & -30.3 & -17.0 & -7.5 & -2.4 \\
\hline
\end{tabular}

supinated position, and forearm pronation from fully supinated position to a position with the palm of the hand on the table. The individual was asked to perform these movements at durations of approximately $0.5,1$, and $2 \mathrm{~s}$.

On real data it is not possible to calculate the MRI measures in (12) and (13), since the volitional EMG cannot be separated from the recorded signal and processed alone through the filters. Instead, the power reduction PR is defined as the ratio of the input power to the output power

$$
\mathrm{PR}=10 \log \frac{\sum x(i)^{2}}{\sum y(i)^{2}} \text {. }
$$

For a perfect filtering PR approaches the ratio of the power of the recorded signal to the power of the volitional EMG. The PR depends of the input signal and the values for the filters should only be compared for the same input signal.

\section{RESULTS}

Adaptive filters with $M=1,3$, and 6 have been evaluated and compared with a fixed comb filter with $M=1$ and $b_{1}=1$ on both simulated and real data obtained from both healthy and tetraplegic individuals.

\section{A. Simulation}

The simulated results are summarized in Table I where both $a$ and $\tau$ are exposed to random variations symmetrical around the fixed value. The variations ranged from 0 to $\pm 100 \%$ of the fixed value. Simulations were carried out with variation applied either to the amplitude or to the shape of the muscle responses and with variations to both parameters. Since the filters need to record $M+1$ frames before an optimal prediction can take place, $\mathrm{MRI}_{x}$ and $\mathrm{MRI}_{y}$ are only calculated after a start-up phase of seven frames. On the following figures, only the filterings after the start-up phase are shown. 

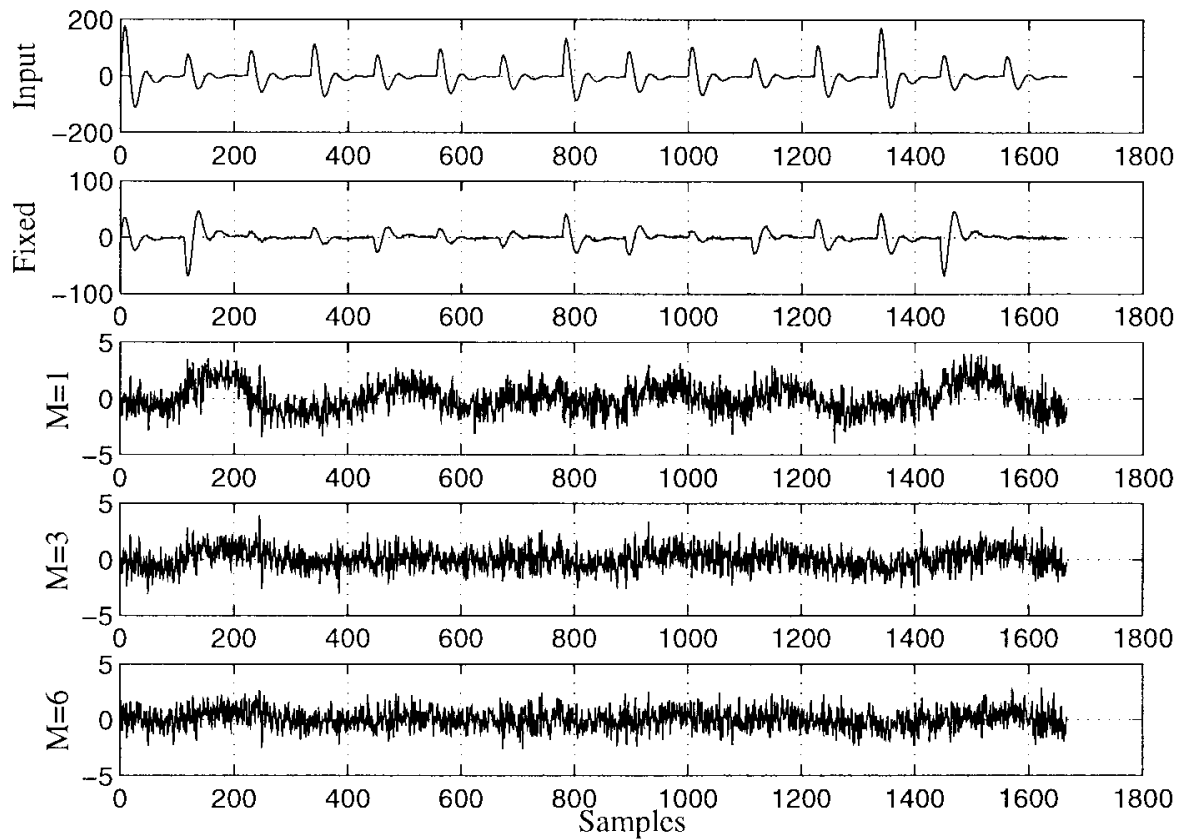

Fig. 7. Simulated results with variation applied to the amplitude of the muscle responses. $a=200 \pm 50 \% . \tau=20 \pm 0 \%$. Arbitrary units on the ordinate.
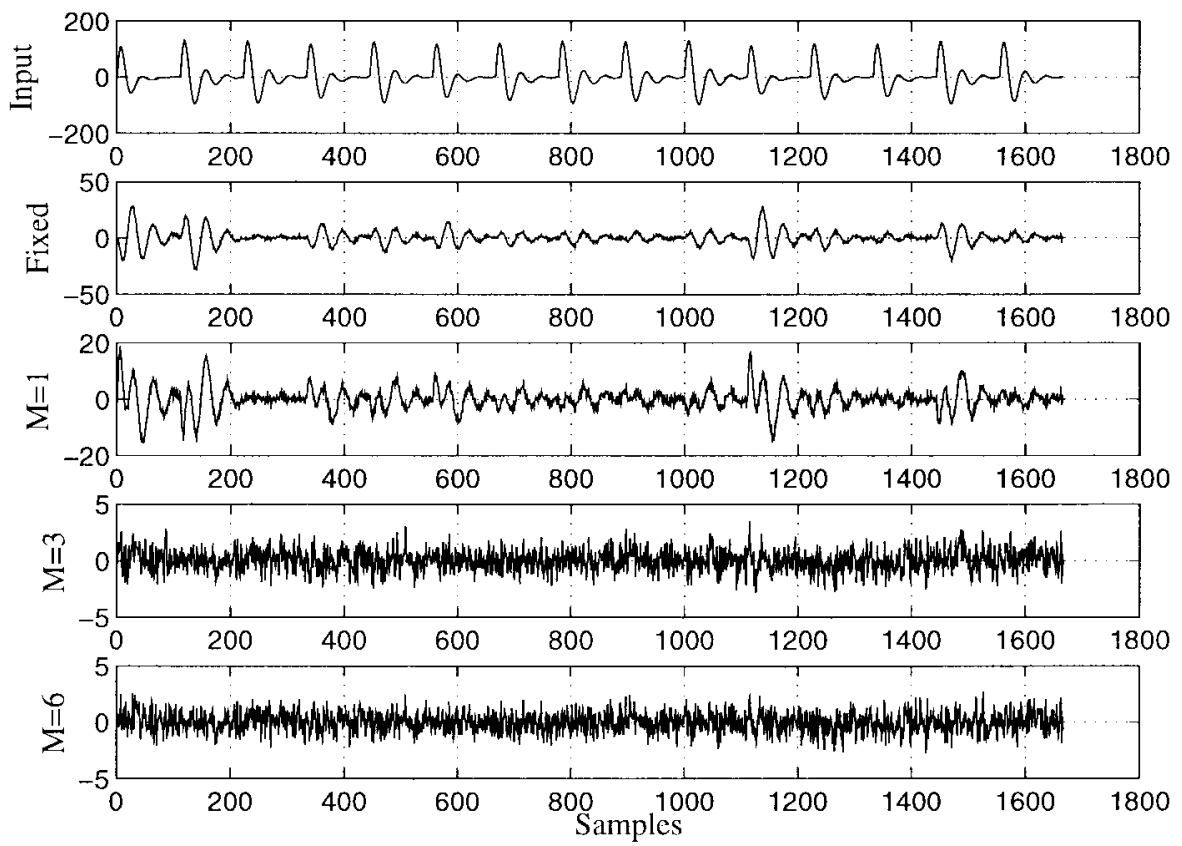

Fig. 8. Simulated results with variation applied to the shape of the muscle responses. $a=200 \pm 0 \% . \tau=20 \pm 40 \%$. Arbitrary units on the ordinate.

When no variation is applied, all the muscle responses are identical. In this case both the fixed and adaptive filters perform a perfect filtering.

When applying variation to the amplitude of the muscle responses, the fixed filter has a poor performance, since it cannot accommodate for the variations, whereas all the adaptive filters show a very good performance even for large variations. An example of amplitude variation of up to $50 \%$ is shown in Fig. 7. Notice that the $M=1$ filter removes most of the muscle responses, but some low-frequency components are still left. An improvement in performance can be obtained by increasing the filter length and for $M=6$ the muscle responses are almost entirely eliminated.

When applying variation to the shape, both the fixed and the $M=1$ adaptive filter show a decreasing performance. But using $M=3$ or $M=6$ adaptive filters still give a good performance even for large variations. An example of the filtering is shown in Fig. 8 with up to $40 \%$ variation in $\tau$. Notice that the $M=3$ and $M=6$ filters both are capable of reducing the muscle response to the same levels as the noise signal, whereas considerable artifacts are still left after the fixed filtering and the $M=1$ adaptive filtering. 

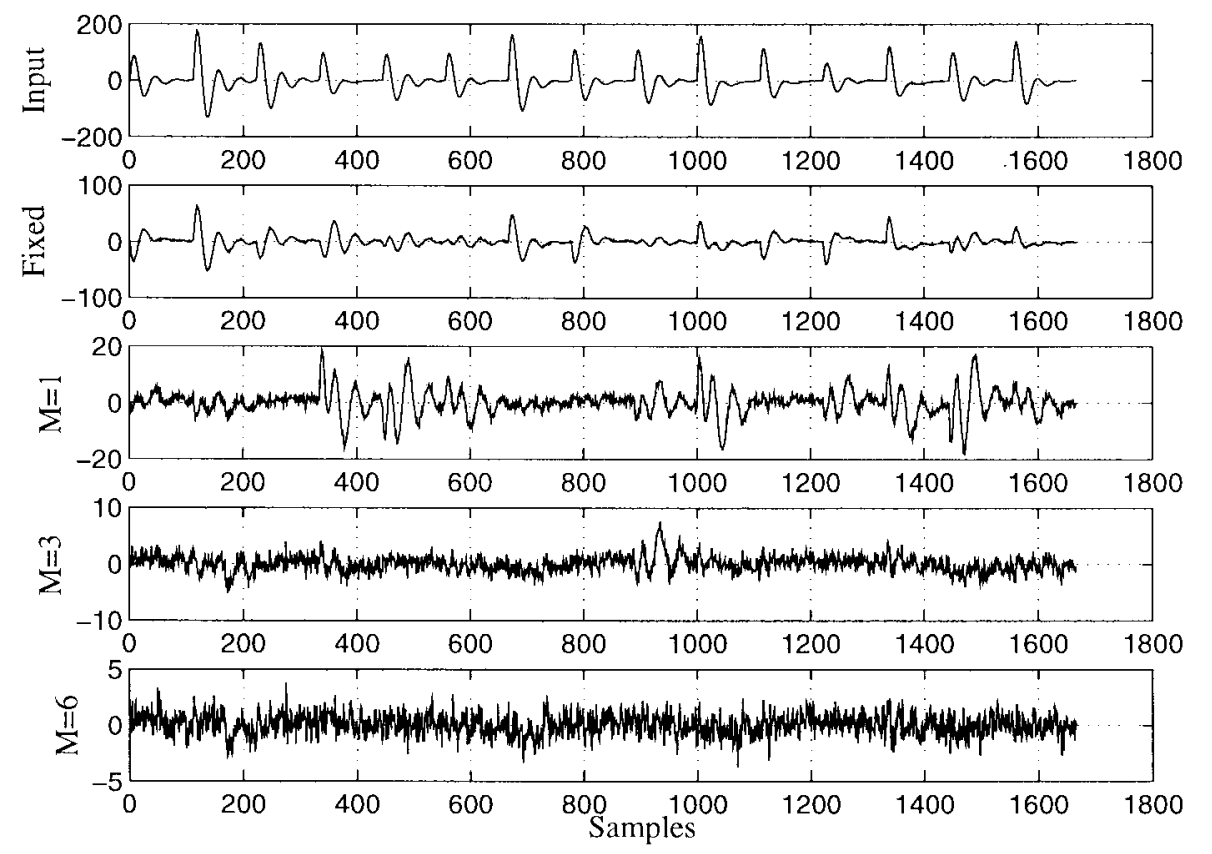

Fig. 9. Simulated results with variations applied to both the amplitude and shape of the muscle responses. $a=200 \pm 50 \% . \tau=20 \pm 100 \%$. Arbitrary units on the ordinate.

Applying simultaneous variations in both amplitude and shape decreases the performance of the $M=3$ adaptive filter, whereas the $M=6$ filter still has a very good performance. Examples of variations of up to $50 \%$ in amplitude together with up to 50\% variation of $\tau$ are shown in Fig. 9. In most cases the $M=3$ filter performs well, but if a muscle response is encountered, which can not be adequately matched by the three previous frames (e.g., from sample 900 to 1000) the filtering will be poor. The $M=6$ filter is still capable of producing a reliable prediction, since it can use up to six previous frames in the prediction.

Notice that even for very large variations in both amplitude and shape an effective filtering can be obtained by using a $M=6$ adaptive filter.

\section{B. Real Data}

The results from the filtering of the real data sets are summarized in Table II. Notice that the $M=1$ adaptive filter performs almost equal as the fixed filter, but in no cases the fixed filter is performing better than the $M=1$ filter. In all the cases an increased power reduction can be obtained by increasing the filter length. For example, using a $M=3$ adaptive filter can give an improvement in PR of up to $8.5 \mathrm{~dB}$ compared with the fixed filter. A further increase in filter length to $M=6$ can give an additional improvement of up to $5.6 \mathrm{~dB}$. The average improvement in power reduction relative to the fixed filter is shown in Fig. 10. For these input sets only a very slight improvement is achieved by using a $M=1$ adaptive filter instead of the fixed filter. But increasing the filter length to $M=3$ gives an average improvement in power reduction of $4.7 \mathrm{~dB}$ and a further increase to $M=6$ gives an average improvement of $7.6 \mathrm{~dB}$.
TABLE II

Power REduction (PR) FROM FILTERING of Real Data. SCI=SPINAl CoRd InJuRED

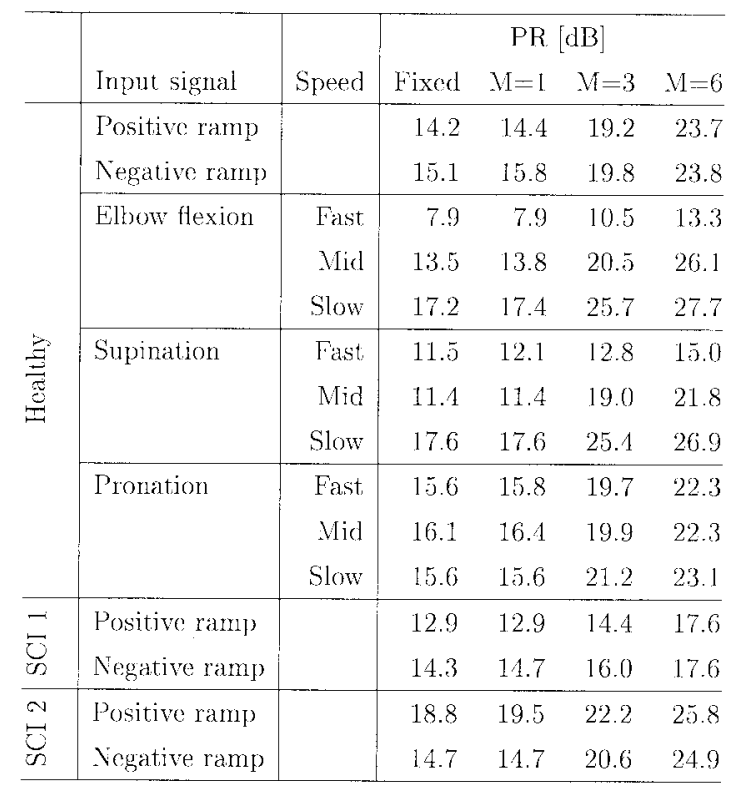

Fig. 11 shows an example where $30 \mathrm{~Hz}$ constant stimulation is applied to a healthy subject, while performing a fast forearm pronation. The fixed and $M=1$ adaptive filters both remove most of the muscle responses, but still in many instances artifacts much larger than the volitional EMG are left in the output signal. Increasing the filter length reduces both the number of artifacts and their amplitude, and for $M=6$ only a few artifacts are slightly larger than the volitional EMG.

An example of filterings from a tetraplegic individual is shown in Fig. 12. A $20-\mathrm{Hz}$ stimulation was applied as a 


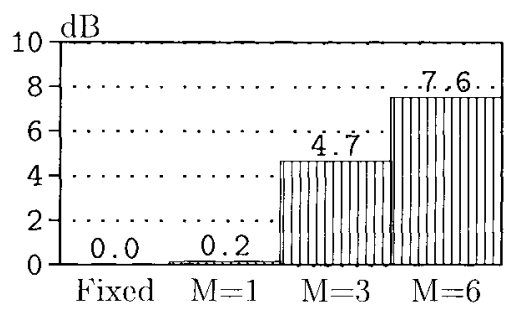

Fig. 10. Average change in power reduction (PR) relative to the fixed filter for filtering of real data.

negative ramp, while the individual was asked to keep his wrist extensor muscle relaxed. Again, a more effective muscle response elimination can be obtained by increasing the filter length. Notice that the $M=6$ adaptive filter in most cases is capable of reducing the muscle response to a level comparable with the background noise. Similar results were obtained by filtering of the three other data sets from tetraplegic individuals, where only occasional artifacts are present in the output signal.

\section{DISCUSSION}

The operation of the adaptive filters are based on scaling previous muscle responses to match the present muscle response optimally. When applying variations to the amplitude of the muscle responses only, a scaling of one previous frame is needed to make a perfect match of the present frame. In theory the adaptive filter performances should be insensitive to variations in amplitude and independent of the filter length. The simulations confirm that the adaptive filters all perform very well even for large variations of the amplitude, and only a small improvement in filter performance can be achieved by increasing the filter length.

When variations are applied to the shape of the muscle responses, the adaptive filters "search" the previous frames to find combinations of muscle responses matching the present muscle response. The chance of success is increased with the number of previous frames to "search" through. Therefore the filter performance will in general increase with increasing filter length. This is demonstrated in both the simulations and in the filtering of real data. In all cases an improved performance can be achieved by increasing the filter length.

If the filtering eliminates all the muscle responses, the output signal will be a weighted average of the volitional EMG's from $M+1$ frames. Therefore increasing the filter length will also increase the time delay of the system. For example stimulating with $20 \mathrm{~Hz}$ and using a $M=6$ adaptive filter will give an averaging time of $350 \mathrm{~ms}$. In order to use the adaptive filters in a practical system, the time delay should not exceed 500 ms [9].

Also the start-up phase is affected by the filter length and will in general have a duration of $M+1$ frames. A special start-up strategy has been implemented to limit the duration of the start-up phase: After recording the first two frames, a $M=1$ adaptive filtering is performed. When a new frame is recorded, the filter length is incremented by one and a $M=2$ adaptive filtering is carried out. This is continued until the final filter length has been reached. By using this strategy the start-up phase can be reduced to two frames (e.g., $100 \mathrm{~ms}$ for $20 \mathrm{~Hz}$ stimulation), but the filtering in this phase will not be optimal since the filter length is reduced.

In Section II-B it was assumed that the volitional EMG signal could be regarded as a band-limited Gaussian noise signal. This is only a good approximation if the number of active motor units is large $(>15)$ [20], [22]. For the muscle activity of a partly paralyzed muscle, this might not be the case. If there exists a cross-correlation between the volitional EMG of the present frame and the volitional EMG of any of the other frames, the adaptive filters might also adapt to this correlation. In this case, the filter will try not only to eliminate the muscle responses, but also the parts of the volitional EMG where the correlation exits. Notice, to avoid this effect it is only required, that the volitional EMG of the regarded frames are mutually uncorrelated, whereas the volitional EMG within a frame can be a correlated signal. In a practical system this effect might reduce the power of the control signal and must be considered.

In a practical situation the choice of filter length will therefore be a compromise of choosing a large enough filter length to obtain a good muscle response suppression and on the other hand to have a noncritical time delay and to avoid adaption to the volitional EMG, and thereby obtain as powerful control signal as possible.

The performance of the adaptive filters are mainly determined by the variations in shape of the muscle responses within the observed $M+1$-frames. During a grasping task several events will contribute to the variations in the muscle responses: changes in muscle length, contraction level and shortening velocity, fatigue and a displacement of the electrodes relative to the muscle. If these changes are slow compared to the adapting rate of the filter (typically, 20-30 $\mathrm{Hz}$ ), the filter accommodates for these changes and the output is unaffected. Occasionally, these variations occur at a rate comparable with the adapting rate. In these cases the present muscle response may be too different in shape from any of the previous $M$ muscle responses. In this situation the adaptive filter is not capable of producing a good result and an artifact will be present in the output signal. An example of this is seen in Fig. 12 where all the filterings leave an artifact in the output signal at app. $700 \mathrm{~ms}$.

It is possible that the stimulation in addition to the muscle response also evokes synchronized reflex activations (e.g., Hwaves). If this activation is synchronized with the stimulation, they will occur identically aligned in each frame of the input signal. In this case the filter will adapt also to these artifacts and try to eliminate them. But since the artifacts must be present in at least two frames before a filtering can occur, an artifact will be present in the output signal the first time the reflex is activated. Reflex activity occuring unsynchronized with the stimulation will cause artifacts in the output signal.

The evaluation of the volitional EMG should be designed, so that it is insensitive to artifacts occurring in the output signal due to inadequate filtering. A solution could be to calculate the 

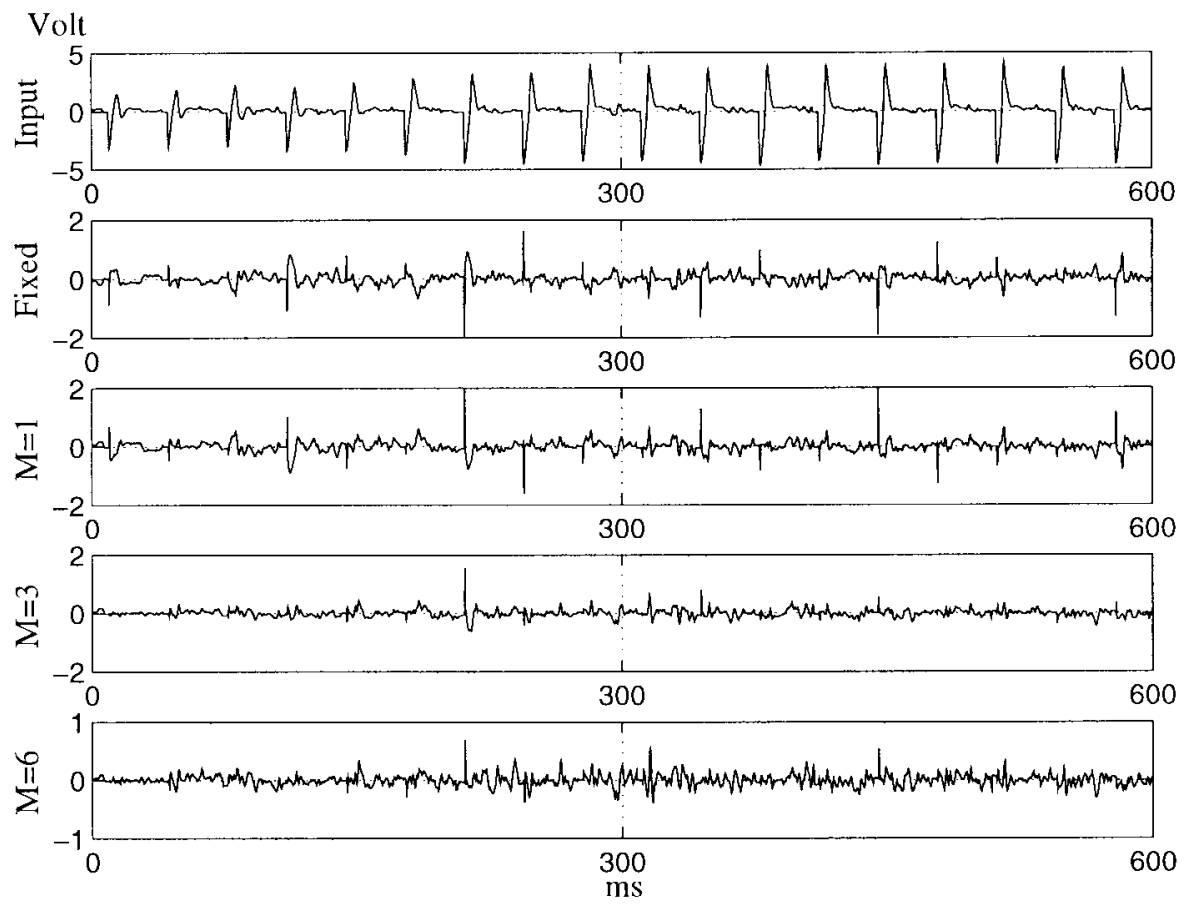

Fig. 11. Example of filtering of a real signal obtained by $30 \mathrm{~Hz}$ constant stimulation of a healthy individual during fast forearm pronation.

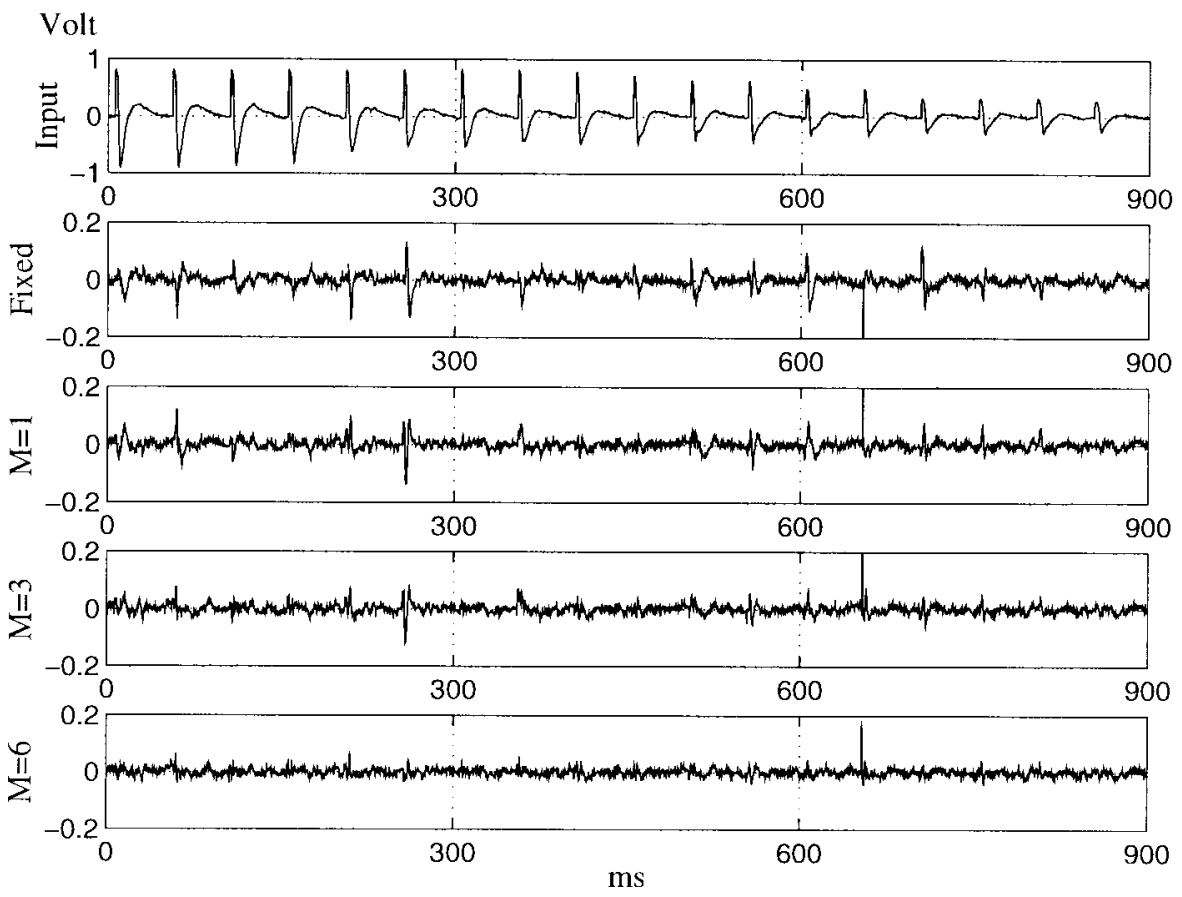

Fig. 12. Example of filtering of a real signal obtained by $20 \mathrm{~Hz}$ stimulation applied as a $3 \mathrm{~s}$ negative ramp on a tetraplegic individual (SCI 1) with no voluntary contraction.

rms-values of the volitional EMG from a number $P^{2}$ previous frames and then use the median rms-value for controlling the stimulation. In this way an inadequate filtering in $(P-1) / 2$ frames can be tolerated, before it will have an effect on the calculation of the stimulation intensity.

${ }^{2} P$ must be an odd number.
A preliminary study has shown that it is possible to use this system to extract the volitional EMG from a partly paralyzed muscle, which is being electrically stimulated, and use it for controlling the stimulation of the same muscle [23]. The type of control which can be realized depends mainly on the strength of the partly paralyzed muscles. For weak muscles only an on/off-control or a simple finite state control might 
be realized, whereas for stronger muscles proportional control is possible.

Even though the neural prosthesis is controlled in a natural way it is still an unnatural task for the user to change the volitional muscle activity when stimulation is present. Especially, relaxation of a stimulated muscle has shown to be difficult and requires special attention from the user. The test individuals in the preliminary study all had the impression that this is a task which is relatively easy learned.

On basis of the data obtained from two tetraplegic subjects, the proposed adaptive filtering technique showed a very promising performance. It was capable of effectively eliminate most of the muscle responses with only occasional artifacts left in the output signal. In order to make a final judgment of the efficiency of the proposed method, the filter should be tested on a larger population of tetraplegic individuals.

\section{CONCLUSION}

To be able to use the volitional EMG as a control signal for the stimulation of the same muscle, the artifacts and the muscle responses caused by the stimulation must be effectively eliminated. The stimulation artifacts are easily removed by a blanking circuit, shutting down the EMG-amplifier at the onset of the stimulation pulses. Due to the duration and the nonstationary nature of the muscle responses also an adaptive filter solution is necessary. Therefore an adaptive linear prediction filter is proposed.

The adaptive filters effectively eliminate the muscle responses and leave a signal with the same power as the volitional EMG. Adaptive filtering for three filter lengths has been tested on both simulated and real data and compared with a conventional fixed comb filter. In general an improved filter performance can be achieved by increasing the filter length. An adaptive filter with length equal to seven stimulation periods were capable of reducing most of the muscle response to a level comparable to the background noise.

Using the blanking circuit and the adaptive filters it is possible to extract the weak volitional EMG from a stimulated partly paralyzed muscle, and use it for controlling the stimulation of the same muscle.

\section{ACKNOWLEDGMENT}

The authors would like to thank the staff and SCI patients at Center for Spinal Cord Injured, University Hospital, Copenhagen, Denmark, for their help and patience during the clinical trials.

\section{REFERENCES}

[1] R. H. Nathan, "An FNS-based system for generating upper limb function in the C4 quadriplegic," Med. Biol. Eng. Comput., vol. 27, pp. 549-556, Nov. 1989.

[2] A. Prochazka and M. Wieler, "The BIONIC glove: Information sheet," Div. of Neurocience, Univ. of Alberta, Edmonton, Alta., T6G 2S2, Tech. Rep., 1994.

[3] N. Hoshimiya, A. Naito, M. Yajima, and Y. Handa, "A multichannel FES system for the restoration of motor functions in high spinal cord injury patients: A respiration-controlled system for the multijoint upper extremity," IEEE Trans. Biomed. Eng., vol. 36, pp. 754-760, July 1989.

[4] P. H. Peckham, E. B. Marsolais, and J. T. Mortimer, "Restoration of a key grip and release in the C6 tetraplegic patient through functional electrical stimulation," J. Hand Surg., vol. 5, no. 5, pp. 462-469, Sept. 1980.
[5] M. W. Keith, P. H. Peckham, G. B. Thrope, K. C. Stroh, B. Smith, J. R. Buckett, K. L. Kilgore, and J. W. Jatich, "Implantable functional neuromuscular stimulation in the tetraplegic hand," J. Hand Surg., vol 14A, no. 3, pp. 524-530, May 1989.

[6] R. Triolo, R. Nathan, Y. Handa, M. Keith, R. R. Betz, S. Carroll, and C. Kantor, "Challenges to clinical deployment of upper limb neuroprosthesis," J. Rehab. Res. Devel., vol. 33, no. 2, pp. 111-122, Apr. 1996.

[7] J. C. Burelbach and P. E. Crago, "Instrumented assessment of FNS hand control during specific manipulation tasks," IEEE Trans. Rehab. Eng., vol. 2, no. 3, pp. 165-176, Sept. 1994.

[8] L. Vodovnik, C. Long, J. B. Reswick, A. Lippay, and D. Starbuck, "Myoelectric control of paralyzed muscles," IEEE Trans. Biomed. Eng., vol. 12, pp. 169-172, July/Oct. 1965.

[9] G. Hefftner, W. Zucchini, and G. G. Jaros, "The electromyogram (EMG) as a control signal for functional neuromuscular stimulation-Part I: Autoregressive modeling as a means of EMG signature discrimination," IEEE Trans. Biomed. Eng., vol. 35, pp. 230-237, Apr. 1988.

[10] G. Hefftner and G. G. Jaros, "The electromyogram (EMG) as a control signal for functional neuromuscular stimulation-Part II: Practical demonstration of the EMG signature discrimination," IEEE Trans. Biomed. Eng., vol. 35, pp. 238-242, Apr. 1988.

[11] M. Solomonow, R. Baratta, H. Shoji, and R. D. D'Ambrosia, "The myoelectric signal of electrically stimulated muscle during recruitment: An inherent feedback parameter for a closed-loop control scheme," IEEE Trans. Biomed. Eng., vol. 33, pp. 735-745, Aug. 1986.

[12] H. J. Holländer, M. Huber, and G. Vossius, "An EMG controlled multichannel stimulator," in Proc. 9th Int. Symp. Adv. Ext. Control Human Extrem., Belgrade, Yugoslavia, 1987, pp. 291-295.

[13] E. -U. Haxthausen, "Restoration of wrist extension using functional electrical stimulation controlled by the remaining voluntary EMG from the stimulated muscles," Ph.D. dissertation, Tech. Univ. Denmark, Electron. Inst., Nov. 1992.

[14] S. Saxena, S. Nikolić, and D. Popović, "An EMG-controlled grasping system for tetraplegics," J. Rehab. Res. Devel., vol. 32, no. 1, pp. 17-24, Feb. 1995.

[15] T. R. D. Scott, K. L. Kilgore, and P. H. Peckham, "Assessment of tristate myoelectric control for bilateral upper extremity neuroprostheses," in Proc. 5th Vienna Int. Workshop Funct. Electrostimulation, Vienna, Austria, Aug. 1995, pp. 343-346.

[16] R. Thorsen, "Elektrisk stimulation af lammede muskler," Master thesis, Tech. Univ. Denmark, Electron. Inst., Jan. 1994.

[17] J. Minzly, J. Mizrahi, N. Hakim, and A. Liberson, "Stimulus artefact suppressor for EMG recording during FES by a constant-current stimulator," Med. Biol. Eng. Comput., vol. 31, pp. 72-75, 1993.

[18] R. Merletti, M. Knaflitz, and C. J. DeLuca, "Electrically evoked myoelectric signals," Crit. Rev. Biomed. Eng., vol. 19, no. 4, pp. 293-340, 1992.

[19] S. Haykin, Adaptive Filter Theory. Englewood Cliffs, NJ: Prentice Hall, 1991.

[20] C. J. DeLuca, "Physiology and mathematics of myoelectric signals," IEEE Trans. Rehab. Eng., vol. 26, pp. 313-325, June 1979.

[21] W. H. Press, B. P. Flannery, S. A Teukolsky, and W. T. Vetterling, Numerical Recipies in $C$. New York: Cambridge University Press, 1988.

[22] N. Hogan and R. W. Mann, "Myoelectric signal processing: Optimal estimation applied to electromyography - part I: Derivation of the optimal myoprocessor," IEEE Trans. Rehab. Eng., vol. 27, pp. 382-395, July 1980.

[23] S. Sennels, R. Thorsen, F. Biering-Sørensen, S. D. Hansen, and O. T. Andersen, "EMG-controlled wrist extension," in Proc. 5th Vienna Int. Workshop on Funct. Electrostimulation, Vienna, Austria, Aug. 1995, pp. 417-420.

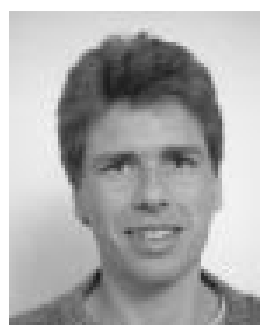

Søren Sennels received the M.Sc. degree in electrical engineering from the Technical University of Denmark, Copenhagen, Denmark, in 1993. He received the $\mathrm{Ph} . \mathrm{D}$. degree in biomedical engineering from the Department of Mathematical Modeling, the Technical University of Denmark, Lyngby, Denmark, concerning rehabilitation of a hand grasp using functional neuromuscular stimulation.

$\mathrm{He}$ is currently employed as a Research Engineer at the Department of Audiology at Gentofte Hospital, Copenhagen, Denmark. His professional interests include digital signal processing, functional neuromuscular stimulation, hardware instrumentation, biomechanics, and biomedical engineering. 


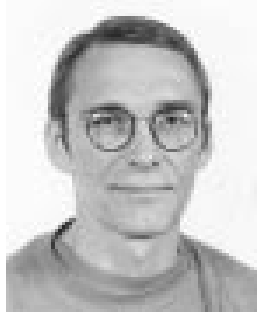

Fin Biering-Sørensen received the M.D. degree from the University of Copenhagen, Copenhagen, Denmark, in 1975. He specialized in physical medicine and rehabilitation in 1982 and rheumatology in 1983. He received the D.M.Sc. degree in low-back pain research in 1984 .

Since 1986, Chief Physician at Department of Medicine and Rehabilitation/Center for Spinal Cord Injured, the Neuroscience Center, Rigshospitalet, Copenhagen University Hospital. His research interests are in the treatment and rehabilitation of spinal cord injured individuals, including functional electrical stimulation. $\mathrm{He}$ has received several national and international research awards. $\mathrm{He}$ is participating in various international projects, committees, and editorial boards.

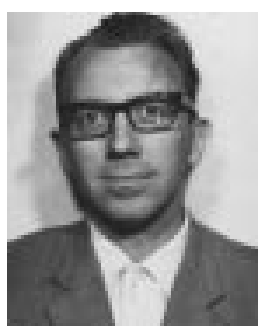

Ole Trier Andersen was born in Denmark in 1930 $\mathrm{He}$ received the M.Sc. degree in electrical engineering and the Ph.D. degree from the Tecnical University of Denmark, Lyngby, in 1956 and 1969, respectively.

From 1956 to 1995, he was with the Electronics Laboratory at the Technical University of Denmark. He is presently Associate Professor at the the Department of Information Technology, and is active in teaching and $\mathrm{Ph} . \mathrm{D}$. student supervision. He has authored textbooks in analog electronics and biomedical engineering, and his biomedical research interests include muscle stimulation, measurements and analysis of movement disturbances, medical applications of ultrasound.
Steffen Duus Hansen (M'83) was born in Copenhagen, Denmark on May 1, 1936. He received the M.Sc. and Ph.D. degrees in electrical engineering from the Tecnical University of Denmark, Lyngby, in 1962 and 1972, respectively.

Early in his professional career, he was engaged in industrial research concerning television systems and measurement equipment. Later, he turned his attention to integrated circuits and aids for the handicapped. $\mathrm{He}$ is now employed at the Technical University of Denmark, Lyngby, as an Associate Professor. His present research interest is digital signal processing for applications in the area of biomedical engineering and speech technology. 